\title{
Self-diagnosed Depression of Chinese High School Students: Do Gender and Urban-Rural Location Make a Difference?
}

\author{
Dilan Luo ${ }^{1, *}$, Jie $\mathbf{Y u}^{2}$ \\ ${ }^{1}$ Wakeland High School, Frisco Independent School District, Frisco, 75033, Texas, United States \\ ${ }^{2}$ College of Educational Science, South China Normal University, 510631, Guangzhou, China
}

Received April 19, 2020; Revised May 27, 2020; Accepted June 23, 2020

Copyright (C2020 by authors, all rights reserved. Authors agree that this article remains permanently open access under the terms of the Creative Commons Attribution License 4.0 International License

\begin{abstract}
Depression is a worldwide common mental illness, and its burden is on the rise globally, particularly for adolescents. Surprisingly, a review of the literature indicates that there are much fewer studies conducted using Chinese adolescents as study subjects, which is not commensurable to China being the fastest developing country with $1 / 5$ of the global population. The purpose of this study is to understand the self-diagnosed depression level of Chinese adolescents and examine if gender and rural-urban location make a difference. Using the Beck Depression Inventory (BDI), 72 high school students in two high schools of different locations in Southern China were surveyed. The survey data were analyzed using descriptive statistics and univariate statistical $t$-tests. Results indicate that the students have on average a mild mood disturbance, while $18 \%$ of them had moderate or severe depression. The mean level of male students' mood disturbance is much higher than that of female students, although it is not statistically significant. Urban students' mood disturbance level is significantly higher than rural students. This study supports the efficient implementation of depression research in China by providing a snapshot of high school students' mental health from the perspective of the surveyed results through self-assessment. Implications for high school mental health counseling and further research are discussed.
\end{abstract}

Keywords High School Students, Depression, Gender, Rural-Urban Location

\section{Introduction}

Depression remains a worldwide common issue leading to serious health problems for adolescents who particularly suffer from its long-lasting and severe intensity, which accounts for $16 \%$ of the global burden of disease and injury in adolescents and remains one of the essential causes of illness and disability among adolescents [1]. The common depressive episodes of adolescents can have various symptoms including stressfulness, anxiety, serious psychological distress, and suicidal tendencies. Moreover, the burden of depression and other mental health disorders is on the rise globally [2]. The increase in adolescent depression can be attributed to the rise of electronic communication, digital media, and less sleep. Particularly, the intensive use of social media by adolescents raises awareness towards mental health issues leading to the significant increase of depression diagnosed adolescents [3].

In the United States, the rates of children aged 3-17 years who have a diagnosed behavior problem, diagnosed anxiety, and diagnosed depression are $7.4 \%, 7.1 \%$, and $3.2 \%$, respectively. Consistent with the growing global trend, the rate of those who have anxiety or depression increased from $5.4 \%$ in 2003 to $8 \%$ in 2007 , and to $8.4 \%$ in 2011-2012 [4]. There is also other research reporting a higher increasing rate of major depressive episodes among adolescents with $13.2 \%$ in 2017 , increasing from $8.7 \%$ in 2005 [5].

The limited prior studies of adolescents' mental health or depression in China seem to point to a much higher rate of mental health illness than in the United States. Using a sample of 2,679 adolescents aged 10-15 years from 25 provinces in China in 2012, Zhou et al [6] reported that $20.3 \%$ of the adolescents' display symptoms of depression [6]. Cheng and Sun [7] revealed that one-third of sample adolescents reported a history of depression using the data early in 2000 [7]. Hesketh and Ding [8] reported a substantially higher prevalence rate of depression among left-behind children ranging from $12.1 \%$ to $51.4 \%$ [8]. 
However, there is research showing a lower rate of depression in Chinese adolescents compared to counterparts in the United States. The 1-year incidence rates of adolescents in China were found to be $2.3 \%$ for unipolar depression, compared to the rate of $10.3 \%$ found in the United States [8-9]. Consistent with the global trend, the same substantial increase in depression rates over recent years in the United States has also been observed in China [10].

Depression as a mental health phenomenon may have been heavily affected by the differences in culture, including the rapid social change present in China within the past 30 years. On the other hand, the inconsistent findings on the adolescents' depression rates may also suggest that mental health issues can be context and time-oriented. Therefore, updated and current studies using different samples in various contexts are needed in better understanding the important issue of adolescents' mental health. In the United States and other western countries, there is a plethora of thorough research conducted on the population of adolescents including studies capturing the generally updated picture of mental health conditions [11], and exploring various factors influencing mental health in adolescents [12]. Surprisingly, a review of the literature reveals that there are much fewer studies conducted using Chinese adolescents as study subjects, which is not commensurable to China being the fastest developing country with one-fifth of the global population.

With mental health being an increasingly global issue for adolescents, there is a paucity of depression research for adolescents in China. The need for better understanding the adolescents' mental health from the research perspective in China is critical since it provides solid evidence for policy and action plans in treating depression in adolescents reducing the risks of poor academic work, quitting school, poor behavior, substance abuse, and poor social functioning.

In order to contribute to the small body of research on mental health in Chinese adolescents, the purpose of this study is to understand the self-diagnosed depression level of Chinese adolescents using a cross-sectional survey study. Seventy-two high school students in two high schools of different locations in Southern China were surveyed using the Beck Depression Inventory (BDI). With the data collected, it was further examined if gender and rural-urban epidemiological location make a difference in Chinese adolescents' mental disorder or depression level.

Gender differences in the overall rate of adolescent depression have been established especially in the United States, with adolescent females exhibiting depressive moods and symptoms more often beginning from early adolescence, leading to a higher rate of diagnosed depression than males in most countries and cultures [13]. A previous study conducted in both an urban and a rural setting of Zhejiang Province, China, found that adolescent girls were more likely to report depression, and those who lived in a rural setting reported the higher vulnerability to the symptoms of anxiety and depression [8]. Children from urban areas have significantly lower rates of depressive symptoms than those in rural areas [6]. Further research pointing to the rapidly changing societal and contextual factors of China is also important in establishing the significance of the factors of adolescent depression in China.

The objective of this study is to better understand the mental health of Chinese adolescents and document research-based evidence related to the possible impact of adolescents' demographics of gender and urbanization on their depression whilst using a self-diagnosis method for students to anonymously report their feelings and conditions more openly. Specifically, this study addresses the following research questions: 1) What is the self-diagnosed depression level of the students in two high schools in China? 2) Is there a difference in the self-diagnosed depression level between the Chinese female and male high school students? 3) Does the location of a Chinese high school student with respect to urbanization make a significant difference on self-diagnosed depression levels?

\section{Methods}

This study used both descriptive and comparative designs in addressing the research questions.

\subsection{Design and Instrument}

The Beck Depression Inventory [14] utilized as the data collection instrument in the cross-sectional survey from the Chinese high school students in two schools. The BDI was previously administered by medical professionals to determine if the potential medical clients need mental treatments or care. It has now become a primary method of self-diagnosis for individual depression screening and research proposes. The BDI is a psychiatric questionnaire widely used for clients or patients to self-report their depression levels. The self-diagnosed depression assessments are an efficient tool to gauge potential depressive disorders without substantial time or cost that are involved in a professional clinical diagnosis for high school students. The results of the BDI, while unable to replace a professional clinical diagnosis, effectively observe the traits of depression and mental health illness.

The BDI focuses on 21 symptom-attitude categories: mood, pessimism, sense of failure, lack of satisfaction, guilty feeling, sense of punishment, self-hate, self-accusations, self-punitive wishes, crying spells, irritability, social withdrawal, indecisiveness, body image, work inhibition, sleep disturbance, fatigability, loss of appetite, weight loss, somatic preoccupation, and loss of libido [14]. These subcategories were chosen to represent 
the intensity of depressive symptoms through clinical observations of depressed and non-depressed psychiatric patients. The BDI questionnaires outline a client's level of depression using their answers scaled by points of 0 to 3 that quantify the severity degree of each depression indicator measures. In interpreting the BDI, the total of above points in each survey item was calculated with the scores of 1) 1-10 presenting ups and downs are considered normal, 2) 11-16: mild mood disturbance, 3) 17-20: borderline clinical depression, 4) 21-30: Moderate depression, 5) 31-40: Severe depression and 6) Over 40: Extreme depression.

With its wide use and thorough validations, the BDI possesses strong validity and reliability. Research studies have consistently revealed solid internal consistency and test-retest reliability of adolescent patients. Acceptable sensitivity and specificity have been shown in the criterion-based validity tests for detecting adolescent depression, supporting its clinical utility as an aid measure for diagnostic purposes [15]. In using the BDI for self-diagnosing Chinese high school students' depression level, the English version was translated into the Chinese version. The Chinese version was reviewed and revised by a professor of psychology from a Chinese university. It was also tested using a small sample of clients in a student counseling center at the university, showing strong face validity and internal validity.

\subsection{Participants, Data Collection, and Statistical Analysis}

The participants of this study are 72 high school students of the 10th and 11th Grade in two schools in Guangdong Province, Southern China. One school is located in an urbanized area in the city of Shenzhen, one of the four largest metropolitan cities in China. The other school is in rural-based Lufeng County. One class of students from each of the above two schools was selected through time convenience as the participants for completing the survey. Of the 72 student respondents, $72.2 \%$ were females; $27.8 \%$ were males. $34.3 \%$ were 17 years old, $50.0 \%$ were 16 , and $15.7 \%$ were 15 .

The approvals of surveying the students at the Chinese high schools were granted by the leaders of the school district, the administration of the high school, and students' parents. Parents were first notified of their children's intention to participate in the survey and were asked to notify the school if they did not agree to their children's survey participation. The 21-question BDI and its self-assessment scale (see Appendix) with accompanying Chinese translation was administered to the two classes of high school students. The BDI surveys were printed and distributed to the students in class after they were informed of the purpose of the study, gave instructions for doing the survey, informed the participants that participation is voluntary, and assured that their responses would be completely anonymous. After completing the surveys, respondents were requested to return their surveys in a prepared sealed envelope. This procedure allows for a secure, anonymous channel for students to report their true feelings.

The survey data collected were analyzed using descriptive statistics and univariate statistical t-tests. Mean scores and standard deviations were calculated to present the self-diagnosed depression levels of high school students. Independent t-tests were used to address the research questions on whether there is a difference in the self-diagnosed depression level between the Chinese male and female high school students and whether the location of a Chinese high school student with respect to urbanization makes a significant difference.

\section{Results}

The overall mean self-diagnosed depression level of the 72 high school students is $M=10.97, S D=6.76$, which is classified at the low end of mild mood disturbance. The numbers and percentages of the high school students by the BDI interpreting scale are presented in Table 1 and Figure 1. The majority of students (56.9\%) self-diagnosed themselves to have normal ups and downs while almost $18 \%$ of the students had moderate or severe depression.

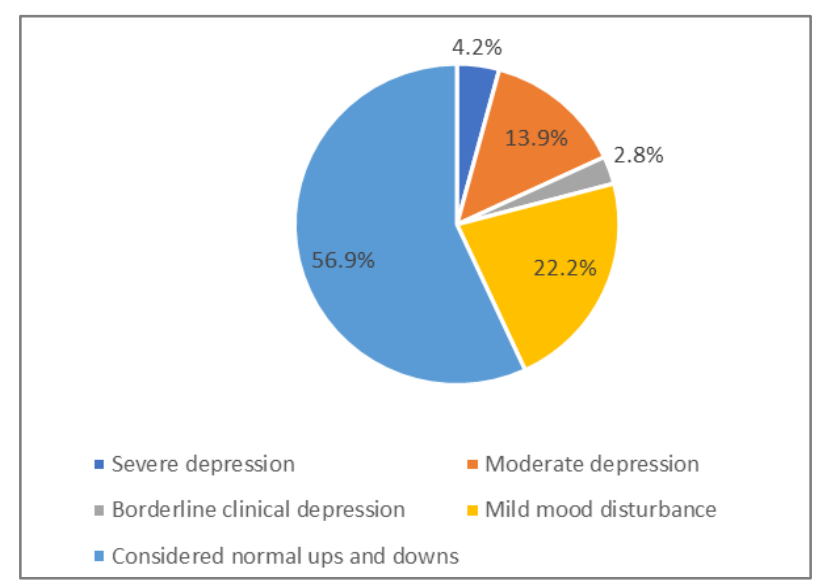

Figure 1. High School Students' Percentage Distribution by the BDI Interpreting Scale

Table 1. Numbers and Percentages of the High School Students by the BDI Interpreting Scale

\begin{tabular}{|c|c|c|c|}
\hline $\begin{array}{c}\text { Total Score } \\
\text { range }\end{array}$ & Interpretation & $n$ & Percentage \\
\hline $0-10$ & $\begin{array}{c}\text { Considered normal ups and } \\
\text { downs }\end{array}$ & 41 & $56.9 \%$ \\
\hline $11-16$ & Mild mood disturbance & 16 & $22.2 \%$ \\
\hline $17-20$ & Borderline clinical depression & 2 & $2.8 \%$ \\
\hline $21-30$ & Moderate depression & 10 & $13.9 \%$ \\
\hline $31-40$ & Severe depression & 3 & $4.2 \%$ \\
\hline $40+$ & Extreme depression & 0 & $0 \%$ \\
\hline
\end{tabular}




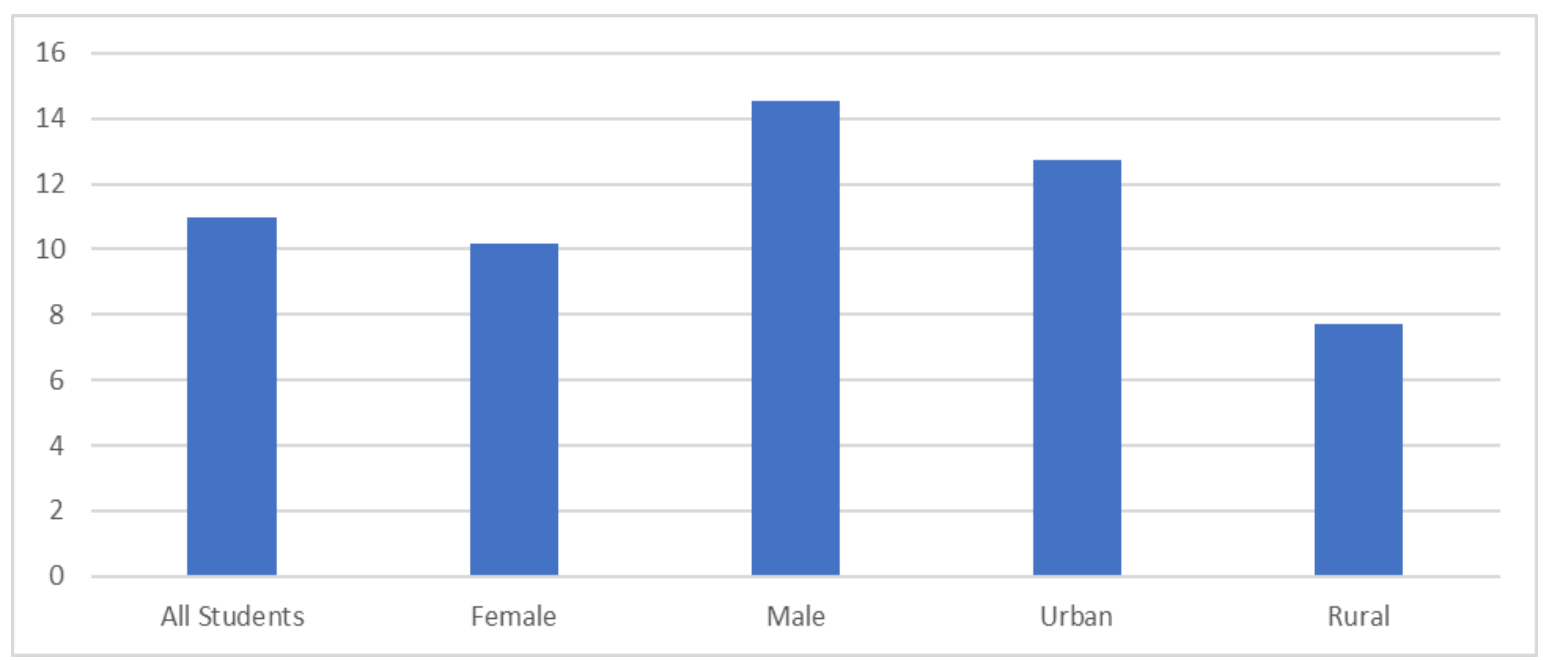

Figure 2. High School Students’ Mean Scores of Self-Diagnosed Mood Disturbance or Depression Level

The mean level of male students' self-diagnosed mood disturbance $(M=14.53, S D=10.63)$ is much higher than that of female students' mean score $(M=10.16, S D=8.37)$. However, the independent $t$-test indicated that there is no significant difference in the mood disturbance level between female and male students $t(70)=1.621, p=.110$, representing a small effect size, $r=0.18$. The other independent $t$-test revealed that urban students' mood disturbance level $(M=12.71, S D=9.61)$ is significantly higher than rural students' mood disturbance level $(M=$ $7.72, S D=6.96), t(70)=2.294, p=.025$, representing a medium-sized effect at $r=0.26$.

\section{Conclusions}

\subsection{Discussion and Conclusions}

The participants of this study are 72 high school students of the 10th and 11th Grade in two schools in Guangdong Province, Southern The internal reliability of the survey items in the Chinese version of the BDI used for data collection was tested with all the participants' survey data. The reliability statistics by SPSS show that Cronbach's alpha for all the survey items is .89, showing a strong internal consistency.

The sample of 72 students from the two high schools surveyed in China has mild mood disturbance on average, while $18 \%$ of them had moderate or severe depression. This finding is consistent with the rate range from $14 \%-23 \%$ of depressive symptoms found in recent previous research using a large sample in China [6]. The rate is higher than the previous research in the United States showing the rate of $13.2 \%$ in major depressive episodes among adolescents was $13.2 \%$ in 2017 [5]. It is even much higher than the rate of $6.2 \%$ for children of 12-17 years old reported by the Center for Disease Control and Prevention [16].
The results of this study support the efficient implementation of adolescent depression research in China by providing a snapshot of high school students' mental health from the perspective of the surveyed results through self-assessment rather than clinical diagnosis. Students with high self-reported BDI scores should seek and receive medical and psychological help that suits their situations. It is important to provide these high school students at school, home, or hospital with the proper options of treatment, clinical care, and therapy that are convenient and effective for them. Students need to be informed, encouraged, or even persuaded to receive psychological counseling and treatments so that they do not have to fight alone in their battle against depression. Those who reported a score of 17 or higher, rating at a level of borderline clinical depression or higher, may need to receive mental health treatments. Psychosocial treatments are effective for mild depression, and while antidepressants can be an effective treatment for moderate-severe depression, they are not the first line of treatment for cases of mild depression. They should not be used for treating depression in children and are not the first line of treatment in adolescents, among whom they are used with extra caution when needed [2].

This study finds that the mean level of male students' mood disturbance is much higher than that of female students, although it does not reach to a statistical significance level or show a strong effect size. However, previous research on medically diagnosed rates of depression in teenagers has presented contrasting evidence [13]. The mood disturbance or depression level of urban students was found to be significantly higher than rural students in this study. Zhou et al. [6] found the opposite, where rates of depressive symptoms are significantly lower in urban areas (14\% of sample children) than in rural areas (23\% of sample children) [6]. The inconsistency between the findings generated by this study and those from previous research seems to show 
that the possible differences in teenagers' mental health by gender and rural-urban location can be situationally, contextually, or even culturally related, which poses a need in conducting research considering such complexity. However, the high or significant difference in the mean level of mood disturbance raises an interesting contrast to the established higher rate of depression found amongst by gender and location. These findings generate important practical implications that the policy or strategies of mental health counseling and treatments need to consider the differentiating characteristics by student gender and school location.

\subsection{Limitations and Future Research}

There are several limitations to this study. First, it presents a sample size of 72 BDI assessments distributed and collected through a survey of two Chinese foreign language high schools. The fairly small sample size coupled with convenience sampling can limit the generalizability of the finding. The small sample size could have also caused difficulty in achieving statistical significance in comparing depression levels between female and male students. It is suggested that a large sample size be used in future research that has better generalizability for testing conclusions. A larger sample size regarding adolescents in the population of a nation allows for a definitive measure of the significance of each factor of depression in adolescents, especially the gender and urbanization factors examined by this present study.

Second, the BDI as used in the study, with its own limitations as a subjective screening tool, has been recommended to be used through multiple screenings and with multiple method assessments [17]. Future research can use multiple assessments of depression coexisting with the BDI, for instance, the revised BDI-II in 1996, which is integrated with the Diagnostic and Statistical Manual of Mental Disorders (DSM-5). Future research if possible should also look to other methods of measuring depression, such as medical diagnosis to observe a medically backed conclusion.

Third, the cross-sectional survey design does not have the capacity in examining the risk factors of depression in the age group of adolescents. Future research is recommended to measure and analyze the risk factors that can potentially impact adolescent depression. These risk factors can include genetic predispositions, the experience of physical, emotional, or sexual abuse, socioeconomic and cultural impacts due to the locality, and cognitive factors of negative thinking and low self-esteem [18]. Research into risk factors affecting depression rates in China is suggested.

\section{REFERENCES}

[1] World Health Organization (2019). Adolescent mental health. Online available at https://www.who.int/news-room/ fact-sheets/detail/adolescent-mental-health.

[2] World Health Organization (2020). Depression. Available at https://www.who.int/newsroom/fact-sheets/detail/depressio n.

[3] K. Saha, J. Torous, S. K. Ernala, C. Rizuto, A. Stafford, M. De Choudhury. A computational study of mental health awareness campaigns on social media. Translational Behavioral Medicine, Vol.9, No.6, 1197-1207.

[4] R. H. Bitsko, J. R. Holbrook, R. M. Ghandour, S. J. Blumberg, S. N. Visser, R. Perou, J. Walkup. Epidemiology and impact of healthcare provider diagnosed anxiety and depression among US children. Journal of Developmental and Behavioral Pediatrics. Vol.39, No.5, 395-403.

[5] J. M. Twenge, A. B. Cooper, T. E. Joiner, M. E. Duffy, S. G. Binau. Age, period, and cohort trends in mood disorder indicators and suicide-related outcomes in a nationally representative dataset. Journal of Abnormal Psychology. Vol.128, No.3, 185-199.

[6] M. Zhou, G. Zhang, S. Rozelle, K. Kenny, H. Xue. Depression symptoms of Chinese children: Prevalence and correlated factors among subgroups. International Journal of Environmental Research and Public Health. Vol.15, No.2, 283.

[7] J. Cheng, Y. H. Sun. Depression and anxiety among left-behind children in China: A systematic review. Child: Care, Health, and Development. Vol.41, No.4, 515-523.

[8] T. Hekseth, Q. J. Ding. Anxiety and Depression in Adolescents in Urban and Rural China. Psychological Reports. Vol.96, No.2, 435-444.

[9] R. C. Kessler. Lifetime and 12-Month Prevalence of DSM-III-R Psychiatric Disorders in the United States. Archives of General Psychiatry. Vol.51, No.1, 8.

[10] A.G. Ryder, J. Sun, X. Zhu, S. Yao, Y. E. Chentsova-Dutton. Depression in China: Integrating Developmental Psychopathology and Cultural-Clinical Psychology. Journal of Clinical Child \& Adolescent Psychology. Vol.41, No.5, 682-694.

[11] Pew Research Center (2019). Most U.S. teens see anxiety and depression as a major problem among their peers. Online available athttps://www.pewsocialtrends.org/2019/0 2/20/most-u-s-teens-see-anxiety-and-depression-as-a-major -problem-among-their-peers/.

[12] H. Steinhausen, C. W. Metzke. Compensatory, Vulnerability, and Protective Factors Influencing Mental Health in Adolescence. Journal of Youth and Adolescence. Vol.30, No.3, 259-280.

[13] B. L. Hankin, R. Mermelstein, L. Roesch. Sex Differences in Adolescent Depression: Stress Exposure and Reactivity Models. Child Development. Vol.78, No.1, 279-295.

[14] A. T. Beck, C. H. Ward, M. Mendelson, J. Mock, J. Erbaugh. An inventory for measuring depression. Archives of General Psychiatry. Vol.4, No.6, 561 
[15] Z. E. Garcia-Batista, K. Guerra-Peña, A. Cano-Vindel, S. X. Herrera-Martinez, L. A. Medrano. Validity and reliability of the Beck Depression Inventory in general and hospital population of Dominican Republic. Vol.13, No.6.

[16] Centers for Disease Control and Prevention (2019). Data and statistics on children's mental health. Online available at https://www.cdc.gov/childrensmentalhealth/data.html.

[17] P. C. Kendall, S. D. Hollon, A. T. Beck, C. L. Hammen, R. E.
Ingram. Issues and recommendations regarding use of the Beck Depression Inventory. Cognitive Therapy and Research. Vol.11, No.3, 289-299.

[18] L. P. Naylor. Depression in adolescence: Depression risk factors, depression effects, depression treatment. Boston Counseling Therapy. Online available at https://thrivebosto n.com/counseling/depression-in-adolescence-depression-ris k-factors-depression-effects-depression-treatment/. 